\title{
Synthesis, antimicrobial activity, pharmacophore modeling and molecular docking studies of new pyrazole-dimedone hybrid architectures
}

\author{
Assem Barakat ${ }^{1,2^{*}}$, Abdullah M. Al-Majid ${ }^{1}$, Bander M. Al-Qahtany ${ }^{1}$, M. Ali ${ }^{1}$, Mohamed Teleb³, \\ Mohamed H. Al-Agamy ${ }^{4,5}$, Sehrish $\mathrm{Naz}^{6}$ and Zaheer Ul-Haq ${ }^{6}$
}

\begin{abstract}
Background: Design and synthesis of pyrazole-dimedone derivatives were described by one-pot multicomponent reaction as new antimicrobial agents. These new molecular framework were synthesized in high yields with a broad substrate scope under benign conditions mediated by diethylamine $\left(\mathrm{NHEt}_{2}\right)$. The molecular structures of the synthesized compounds were assigned based on different spectroscopic techniques ( ${ }^{1} \mathrm{H}-\mathrm{NMR},{ }^{13} \mathrm{C}-\mathrm{NMR}$, IR, MS, and CHN).

Results: The synthesized compounds were evaluated for their antibacterial and antifungal activities against $S$. aureus ATCC 29213, E. faecalis ATCC29212, B. subtilis ATCC 10400, and C. albicans ATCC 2091 using agar Cup plate method. Compound $\mathbf{4 b}$ exhibited the best activity against $B$. subtilis and E. faecalis with $\mathrm{MIC}=16 \mu \mathrm{g} / \mathrm{L}$. Compounds $\mathbf{4 e}$ and $\mathbf{4 I}$ exhibited the best activity against S. aureus with MIC $=16 \mu \mathrm{g} / \mathrm{L}$. Compound $\mathbf{4 k}$ exhibited the best activity against $B$. subtilis with $\mathrm{MIC}=8 \mu \mathrm{g} / \mathrm{L}$. Compounds $\mathbf{4 0}$ was the most active compounds against C. albicans with MIC $=4 \mu \mathrm{g} / \mathrm{L}$.

Conclusion: In-silico predictions were utilized to investigate the structure activity relationship of all the newly synthesized antimicrobial compounds. In this regard, a ligand-based pharmacophore model was developed highlighting the key features required for general antimicrobial activity. While the molecular docking was carried out to predict the most probable inhibition and binding mechanisms of these antibacterial and antifungal agents using the MOE docking suite against few reported target proteins.
\end{abstract}

Keywords: Pyrazole, Dimedone, Antifungal activity, Antimicrobial activity, Structure activity relationship, Inhibition mechanism prediction

\section{Background}

Nosocomial infections caused by antibiotic-resistant gram-positive bacteria have become a serious medical problem with an alarming increasing rate worldwide. Methicillin-resistant Staphylococcus aureus (MRSA), vancomycin-resistant enterococci (VRE) and penicillin-resistant Streptococcus pneumoniae (PRSP) are of particular concern among various hospital-acquired

\footnotetext{
*Correspondence: ambarakat@ksu.edu.sa

${ }^{1}$ Department of Chemistry, Faculty of Science, King Saud University, P. O. Box 2455, Riyadh 11451, Saudi Arabia

Full list of author information is available at the end of the article
}

infections [1]. Accordingly, emerging investigations have provided new insights into developing novel, safe and effective antibacterial agents. Within this scope, pyrazole based antibacterial agents attracted great interest [2]. Generally, pyrazoles display innumerable pharmacological activities ranging from analgesic, antipyretic, antimicrobial, anti-inflammatory, anticancer effects to antidepressant, anticonvulsant, and selective enzyme inhibitory activities [2-11]. Recently, Barakat et al, have been reported novel pyrazole hybrid architectures as efficient antibacterial agents. Various pharmacophores were linked to the pyrazole core to build bioactive scaffolds [12, 13]. Within this approach, cyclic dicarbonyl 
compounds of the type dimedone have attracted our interest. Dimedone has been utilized successfully as pharmacophoric building block in various antimicrobial agents such as xanthenes $[14,15]$, substituted chromenes [16], macrocyclic metal complexes [17], quinazoline derivatives [18], tetrahydro quinolone diones [19] and acridine based compounds [20]. Recognizing these facts and in continuation of our previous work $[12,13]$ new hybrid molecules incorporating pyrazoles and dimedone in a single molecular framework were designed and synthesized. We subjected our target compounds to pharmacophore modeling and molecular docking on different target proteins to explore their mode of action.

\section{Results and discussion}

\section{Chemistry}

The designed bioactive scaffolds were synthesized utilizing green approach. The pyrazole-dimedone derivatives were prepared as shown in Scheme 1 via one pot Knoevenagel condensation Michael addition of 3-methyl-1-phenyl-1 $H$-pyrazol-5(4H)-one, 1,3-dicarbonyl compound (dimedone) and various aldehydes mediated by aqueous $\mathrm{NHEt}_{2}$. This one pot multicomponent reaction afforded the final targets as hybrid frameworks $4 \mathbf{a}-\mathbf{o}$ in good yields $(40-78 \%)$ with substrate tolerance of pyrazoledimedone derivatives. The chemical structures of all the synthesized compounds were assigned by the aid of physical and spectroscopic methods $\left({ }^{1} \mathrm{H}-\mathrm{NMR},{ }^{13} \mathrm{C}-\mathrm{NMR}, \mathrm{IR}\right.$, and elemental analyses).

The suggested mechanisms for obtaining the target compounds are shown in Scheme 2. Olefin is formed by Knoevenagel condensation of aryl aldehyde $\mathbf{1}$ and 1,3-diketone 2 to give benzylidenecyclohexandione intermediate which acts as a Michael acceptor. This Michael acceptor is attached by 3-methyl-1-phenyl-1 $H$-pyrazol$5(4 H)$-one 3 (Michael donor) to give the requisite final targets 4a (Path A). Another bath way is Knoevenagel condensation between aryl aldehyde $\mathbf{1}$ and 3-methyl1-phenyl-1H-pyrazol-5(4H)-one $\mathbf{3}$ to generate benzylidenepyrazolone intermediate which acts as a Michael acceptor. This Michael acceptor is attacked by 1,3-diketone 2 (Michael donor) to afford the final product $\mathbf{4 a}$ (Path B).

\section{Antimicrobial activity}

The synthesized pyrazole-dimedone derivatives showed various antibacterial activities. Results of the bactericidal activity are shown in Table 1; the minimum inhibitory concentration (MIC) results are expressed as $\mu \mathrm{g} / \mathrm{L}$ inhibition.

\section{Antibacterial activity against gram positive bacteria}

The antibacterial activity of the novel pyrazole-dimedone compounds were evaluated against gram positive bacteria including E. faecalis ATCC29212, S. aureus ATCC 29213, and B. subtilis ATCC 10400. Ciprofloxacin was used as standard drug.

The results listed in Table 1 revealed that all pyrazoledimedone compounds were active against the testedstrains including S. aureus, E. faecalis, and B. subtilis. Pyrazole-dimedone $\mathbf{4 k}$ was the most active compound against $B$. subtilis with MIC value of $8 \mu \mathrm{g} / \mathrm{L}$. Compounds 4e and 41 having 3-methyl and 4-trifluromethyl substituents on the phenyl ring respectively exhibited good activity against $S$. aureus with MIC value of $16 \mu \mathrm{g} / \mathrm{L}$. Compounds $\mathbf{4 a - d}, \mathbf{4 f}, \mathbf{g , \mathbf { i } , \mathbf { k }}$ and $\mathbf{4 m - \mathbf { o }}$ showed relatively lower activity against $S$. aureus with MIC value of $32 \mu \mathrm{g} / \mathrm{L}$. Compounds $\mathbf{4 h}$ and $\mathbf{4 j}$ having 4-nitro and 4-methoxy substituents on the phenyl ring were the least active derivatives against $S$. aureus with MIC values of $64 \mu \mathrm{g} / \mathrm{L}$. Compound $4 \mathrm{~b}$ bearing unsubstituted phenyl ring exhibited good activity against $E$. faecalis with MIC values of $16 \mu \mathrm{g} / \mathrm{L}$. Compounds $4 \mathbf{a}, \mathbf{c}-\mathbf{e}, \mathbf{4 g}, \mathbf{h}$ and $\mathbf{4 j - o}$ showed lower activity against $E$. faecalis with MIC value of $32 \mu \mathrm{g} / \mathrm{L}$. Compounds $4 \mathbf{f}$ and $4 \mathbf{i}$ having 4-bromo and 3-nitro substituents on the phenyl ring respectively were shown as the least active derivatives against $E$. faecalis with MIC value of $64 \mu \mathrm{g} / \mathrm{L}$.

Substituted pyrazole-dimedone $\mathbf{4 b}$ without substituent on the phenyl ring and 4o having thiophene ring exhibited good activity against $B$. subtilis with MIC value of $16 \mu \mathrm{g} / \mathrm{L}$. Compounds $\mathbf{4 a}, \mathbf{c}, \mathbf{d}, \mathbf{4 f}-\mathbf{j}$ and $\mathbf{4 l - o}$ showed lower activity against $B$. subtilis with MIC value of $32 \mu \mathrm{g} / \mathrm{L}$. Compound $4 \mathbf{e}$ having 3-methyl substituent on the phenyl ring was shown to be the least active against B. subtilis with MIC value of $64 \mu \mathrm{g} / \mathrm{L}$.

\section{Antifungal activity}

The newly synthesized pyrazole-dimedone derivatives were evaluated for their antifungal activity against fungi C. albicans (ATCC 2091) by the diffusion agar and serial dilution method (BSAC, 2015) [23] Fluconazole was used as standard antifungal agent. Results shown in Table 1 revealed that all pyrazole-dimedone compounds 4a-o were active against the tested-strains $C$. albicans ATCC 2091. Pyrazole-dimedone 40 bearing thiophene was the most active compounds from this series against $C$. albicans ATCC 2091 with MIC value of $4 \mu \mathrm{g} / \mathrm{L}$. Compounds $\mathbf{4 c}, \mathbf{d}, \mathbf{h}, \mathbf{k}, \mathbf{m}$ possessed good activity against $C$. albicans with MIC values of $16 \mu \mathrm{g} / \mathrm{L}$. Compounds $4 \mathbf{a}, \mathbf{b}, \mathbf{4 e - g}$, and $4 \mathbf{i}, \mathbf{j}, \mathbf{g}, \mathbf{n}$ were the least active among this series as antifungal agent with MIC values of $32 \mu \mathrm{g} / \mathrm{L}$. 


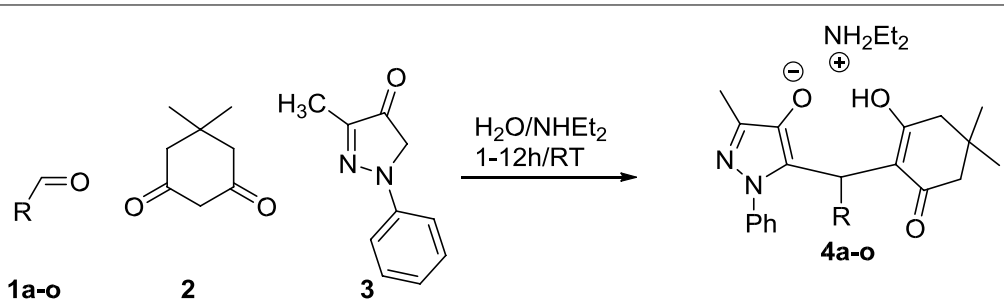

\begin{tabular}{|c|c|c|c|}
\hline$\#$ & 4 & $\mathbf{R}$ & yield $(\%)^{b}$ \\
\hline 1 & $4 \mathbf{a}$ & $2,4-\mathrm{Cl}_{2} \mathrm{Ph}$ & 78 \\
\hline 2 & $4 b$ & $\mathrm{Ph}$ & 62 \\
\hline 3 & $4 c$ & $p$-ClPh & 50 \\
\hline 4 & $4 d$ & $p-\mathrm{CH}_{3} \mathrm{Ph}$ & 62 \\
\hline 5 & $4 e$ & $m-\mathrm{CH}_{3} \mathrm{Ph}$ & 66 \\
\hline 6 & $4 f$ & $p$-BrPh & 71 \\
\hline 7 & $4 \mathrm{~g}$ & $m$-BrPh & 70 \\
\hline 8 & $4 h$ & $p-\mathrm{NO}_{2} \mathrm{Ph}$ & 52 \\
\hline 9 & $4 \mathbf{i}$ & $m-\mathrm{NO}_{2} \mathrm{Ph}$ & 63 \\
\hline 10 & $4 \mathbf{j}$ & $p-\mathrm{CH}_{3} \mathrm{OPh}$ & 64 \\
\hline 11 & $4 \mathbf{k}$ & $p$-FPh & 57 \\
\hline 12 & 41 & $p-\mathrm{CF}_{3} \mathrm{Ph}$ & 76 \\
\hline 13 & $4 m$ & $2,6-\mathrm{Cl}_{2} \mathrm{Ph}$ & 40 \\
\hline 14 & $4 n$ & 2-Naphthaldehyde & 76 \\
\hline 15 & 40 & Thiophene & 75 \\
\hline
\end{tabular}

${ }^{a}$ All reactions were carried out with aldehyde 1 (1.5 mmol), 5,5-dimethylcyclohexane1,3-dione 2 (1.5 mmol), 3-methyl-1-phenyl-1H-pyrazol-5(4H)-one $(1.5 \mathrm{mmol})$ and amine $(1.5 \mathrm{mmol})$ in water $(1.5 \mathrm{~mL})$ for the specified time. ${ }^{\mathrm{b}}$ Yield of isolated product. 


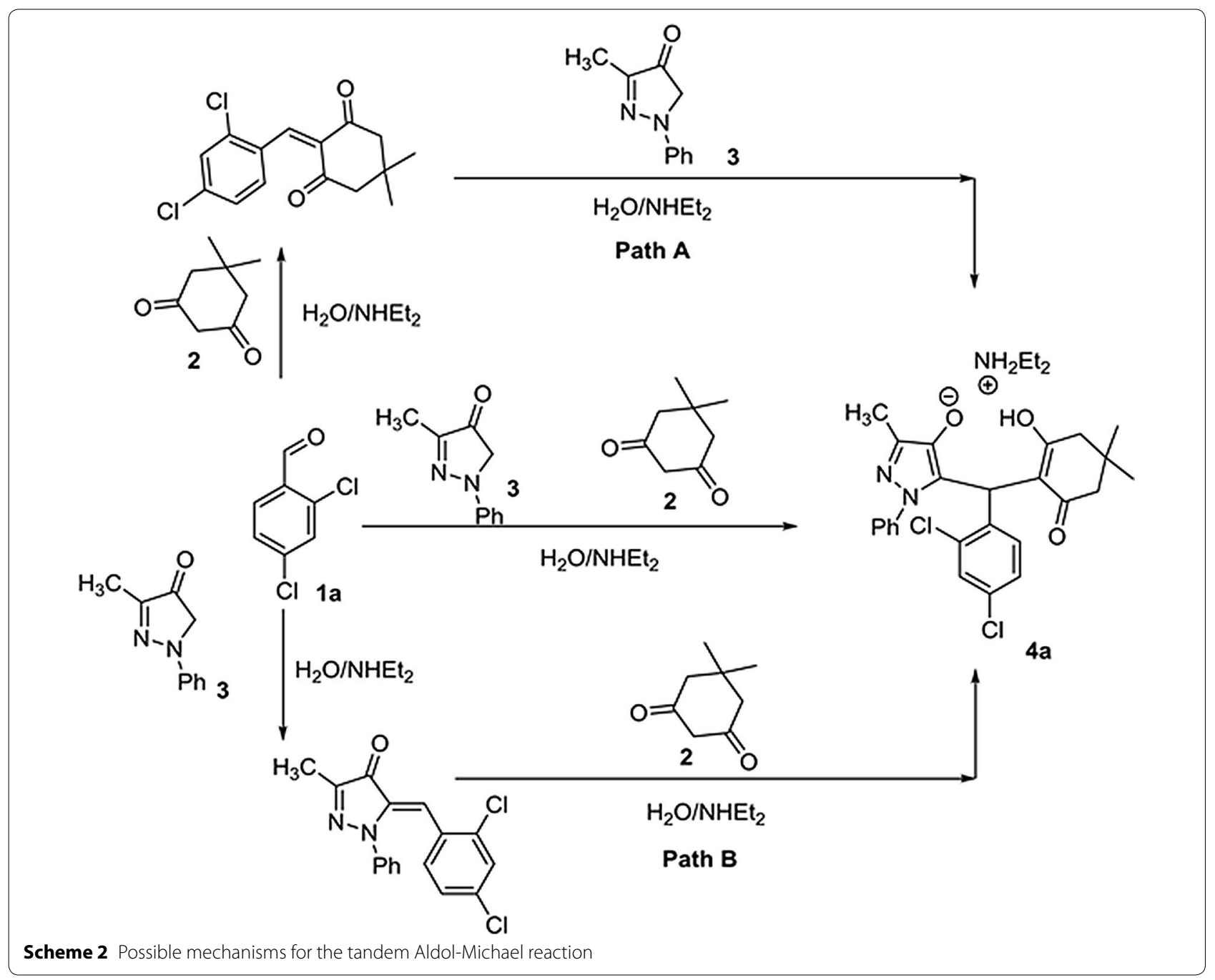

Structure activity relationship profiling via pharmacophore modeling

First of all, to predict the structure activity relationship (SAR) of all the newly synthesized antimicrobial compounds, a ligand-based pharmacophore model was developed. This is the most reliable way to design new potent active molecules having similar scaffolds by utilizing their biological data in computational predictions. In this study, the selected pharmacophore including one hydrogen bond acceptor (F1: Acc\& ML), one hydrogen bond donor (F2: Don, Acc\& ML) and one hydrophobic feature with an aromatic center (F3: ML/Hyd/Aro) (Fig. 1a) was mapped over active compounds (Fig. 1b). The mapping was evaluated on the basis of their lowest RMSD between query and matching annotations (Fig. 1c, d).

The lowest RMSD indicates better compound fitness to the selected model. Results in Table 2 showed that all the active compounds were able to satisfy the pharmacophoric features of the generated model with RMSD values ranging from 0.3907 to $0.6571 \AA$ along with their most suitable alignment of each compound over query. These results indicated the critical role of aromatic ring substitution which greatly effects the spatial orientation of cyclohexane ring with respect to the pyrazole moiety. This might be the best explanation to understand the differences in their respective antimicrobial activity profile.

\section{Docking simulation to predict the mode of inhibition}

After SAR profiling, docking studies were carried out to predict the most suitable binding pose and inhibition mechanism of newly synthesized derivatives. But before docking, based on the principle that similar Compounds tend to bind to the same proteins, we predicted few protein targets reported against reference compounds (ciprofloxacin and fluconazole) and docked our active compounds against them. Binding DB brought in seven different target proteins i.e. Dihydrofolate Reductase (DHFR) (PDB ID 4HOF), Secreted Aspartic Protease 
Table 1 Results of cup-plate method expressed as minimum inhibitory concentrations (MIC) of the compounds in ( $\mu \mathrm{g} / \mathrm{L}$ )

\begin{tabular}{|c|c|c|c|c|c|c|c|c|c|}
\hline \multirow[t]{3}{*}{ Entry } & \multirow[t]{3}{*}{ Compounds } & \multicolumn{6}{|c|}{ Gram positive bacteria } & \multirow{2}{*}{\multicolumn{2}{|c|}{$\begin{array}{l}\text { Yeast } \\
\text { C. albicans ATCC } \\
2091\end{array}$}} \\
\hline & & \multicolumn{2}{|c|}{$\begin{array}{l}\text { S. aureus } \\
\text { ATCC } 29213\end{array}$} & \multicolumn{2}{|l|}{$\begin{array}{l}\text { E. faecalis } \\
\text { ATCC29212 }\end{array}$} & \multicolumn{2}{|l|}{$\begin{array}{l}\text { B. subtilis } \\
\text { ATCC10400 }\end{array}$} & & \\
\hline & & $\mathrm{CPM}(\mathrm{mm})$ & $\mathrm{MIC}(\mu \mathrm{g} / \mathrm{L})$ & $\mathrm{CPM}(\mathrm{mm})$ & $\mathrm{MIC}(\mu \mathrm{g} / \mathrm{L})$ & $\mathrm{CPM}(\mathrm{mm})$ & MIC $(\mu \mathrm{g} / \mathrm{L})$ & $\mathrm{CPM}(\mathrm{mm})$ & $\mathrm{MIC}(\mu \mathrm{g} / \mathrm{L})$ \\
\hline 1 & $4 a$ & 13 & 32 & 14 & 32 & 12 & 32 & 14 & 32 \\
\hline 2 & $4 b$ & 15 & 32 & 13 & 16 & 15 & 16 & 15 & 32 \\
\hline 3 & $4 c$ & 13 & 32 & 24 & 32 & 16 & 32 & 15 & 16 \\
\hline 4 & $4 d$ & 16 & 32 & 16 & 32 & 18 & 32 & 16 & 16 \\
\hline 5 & $4 e$ & 19 & 16 & 15 & 32 & 14 & 64 & 14 & 32 \\
\hline 6 & $4 f$ & 14 & 32 & 13 & 64 & 15 & 32 & 14 & 32 \\
\hline 7 & $4 g$ & 14 & 32 & 15 & 32 & 16 & 32 & 14 & 32 \\
\hline 8 & $4 h$ & 12 & 64 & 14 & 32 & 16 & 32 & 17 & 16 \\
\hline 9 & $4 i$ & 14 & 32 & 12 & 64 & 17 & 32 & 14 & 32 \\
\hline 10 & $4 j$ & 10 & 64 & 13 & 32 & 10 & 32 & 13 & 32 \\
\hline 11 & $4 k$ & 13 & 32 & 13 & 32 & 20 & 8 & 15 & 16 \\
\hline 12 & 41 & 16 & 16 & 16 & 32 & 16 & 32 & 14 & 32 \\
\hline 13 & $4 m$ & 15 & 32 & 13 & 32 & 12 & 32 & 16 & 16 \\
\hline 14 & $4 n$ & 14 & 32 & 13 & 32 & 15 & 32 & 14 & 32 \\
\hline 15 & 40 & 13 & 32 & 20 & 32 & 15 & 16 & 21 & 4 \\
\hline \multirow[t]{2}{*}{ STD } & Ciprofloxacin & 27 & $\leq 0.25$ & 24 & $\leq 0.25$ & 25 & $\leq 0.25$ & ND & ND \\
\hline & Fluconazole & ND & ND & ND & ND & ND & ND & 28 & 0.5 \\
\hline
\end{tabular}

(PDB ID 3Q70), and $N$-myristoyl Transferase (PDB ID 1IYL) from $C$. Albicans as fungal target together with Dihydrofolate Reductase (PDB ID 3FYV), Gyrase B (PDB ID 4URM), Thymidylate Kinase (TMK) (PDB ID 4QGG) and Sortase A (PDB ID 2MLM) from S. aureus as bacterial target. Among all these seven proteins, only two proteins i.e. one proteins (Thymidylate Kinase) from $S$. aureus [21] and one protein ( $N$-myristoyl transferase) from C. albican [22] presented good binding affinity, while all other targets showed very few or no interactions with these derivatives.

The potencies of these newly synthesised derivatives were measured computationally in terms of their dock Scores. Dock score which is actually the strength of the non-covalent interactions among multiple molecules within the binding pocket of a target protein. The more negative the score is, the more favorable interactions between compound and the target protein are. Here in our study, the compound $4 \mathbf{l}$ being the most potent antibacterial agent against TMK (ID: 4QGG) from S. aurues, displayed the highest negative score of $-6.86 \mathrm{kcal} / \mathrm{mol}$ which is comparable of the standard drug ciprofloxacin with the score of $-6.9 \mathrm{kcal} / \mathrm{mol}$. Similarly, $4 \mathrm{o}$ being the most potent antifungal agent displayed good docking score of $-8.7 \mathrm{kcal} / \mathrm{mol}$ and molecular interactions with $N$-myristoyl transferase (NMT) enzyme from $C$. Albicans.
Among all derivatives, compound 41 displayed the same electrostatic and hydrophobic interactions with crucial residues of TMK protein from $S$. aureusas presented by co-crystallized ligand. As illustrated in Fig. 2, the substituted part of compound $\mathbf{4 1}$ moved inside the cavity where both chlorine atoms at 2 and 4 positions were engaged in the formation of two halogen bonds with the amino groups of Arg70 and Gln101 at $2.14 \AA$ and $2.53 \AA$, respectively. Moreover, dichloro substituted benzene ring along with the pyrazole ring displayed various $\pi-\pi$ and $\pi$-cation interactions with the crucial residues Phe66 and Arg92 of the target protein. Apart from it, the carbon atom located at $\mathrm{R}$ position and methyl of pyrazole ring were observed to establish hydrophobic interactions with Arg48 and Phe66 of TMK protein that might be responsible for their potent antibacterial activity.

Comparatively, compound $\mathbf{4 k}$ being the most active against $B$. subtilis species showed less or very few interactions with the TMK protein (4QGG) from $S$. aureus origin (Fig. 3).

Similarly, the molecular visualization of 40 revealed a number of significant electrostatic and hydrophobic interactions with the crucial residues of NMT. Figure 4 showed that the hydroxyl moiety attached at dimedone ring presented visible hydrogen bond with Tyr107 at a distance of $2.48 \AA$. Apart from it, three $\pi-\pi$ interactions were observed among phenyl and thiol and 

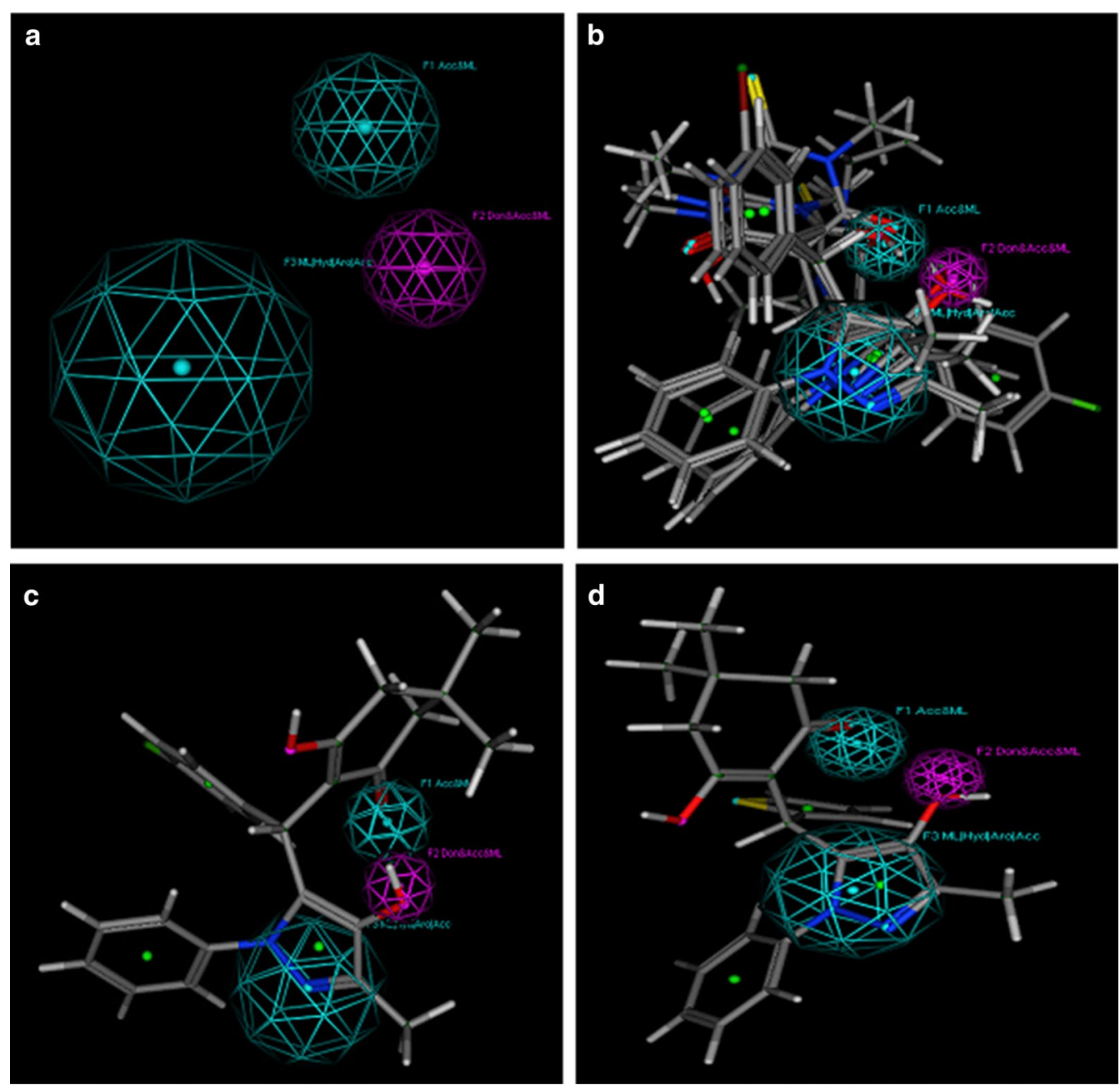

Fig. 1 a Best query displaying pharmacophoric features shared by active lead compounds as colored spheres (cyan for hydrogen bond acceptor function with metal ligator (F1: Acc\& ML), pink for hydrogen bond acceptor/donor function with metal ligator (F2: Don, Acc\& ML) as well as cyan for hydrophobic region with aromatic centre, hydrogen bond acceptor or metal ligator function (F3: ML/Hyd/Aro/Acc). $\mathbf{b}$ Validation of the selected query; mapping of previously reported active compounds $\mathbf{4 a}$ and $\mathbf{4 n}$ [12] as well as $\mathbf{4 a}$ and $\mathbf{4 f}$ [13], showing RMSD values in acceptable range (0.2823-0.4993). c Mapping of compound $\mathbf{4 k}$ on pharmacophore model. $\mathbf{d}$ Mapping of compound $\mathbf{4 0}$ on pharmacophore model

Table 2 RMSD values along with their suitable alignment for Hit Compounds

\begin{tabular}{llllllllll}
\hline Comp. no. & 4b & 4c & $\mathbf{4 d}$ & $\mathbf{4 e}$ & $\mathbf{4 h}$ & $\mathbf{4 k}$ & $\mathbf{4 l}$ & $\mathbf{4 m}$ & $\mathbf{4 0}$ \\
\hline $\operatorname{RMSD}(\AA)$ & 0.3907 & 0.4715 & 0.4639 & 0.4663 & 0.4662 & 0.5938 & 0.5070 & 0.6571 & 0.5660 \\
\hline
\end{tabular}

hotspot residues Phe117, Tyr225 and Tyr 354. Simultaneously, several hydrophobic interactions were also noticed among compound 40 and the crucial residues i.e. Tyr107, Phe 117, Tyr119, Tyr225, Tyr335. These results predicted TMK (S. aureus) and NMT (C. albicans) as the most probable targets for the antibacterial and antifungal activity of these newly synthesized agents.

\section{Conclusions}

By using one-pot green protocol a series of pyrazoledimedone derivatives $(\mathbf{4 a}-\mathbf{o})$ were synthesized in high yields with a broad substrate scope under mild reaction conditions in water mediated by $\mathrm{NHEt}_{2}$. The requisite compounds were evaluated for their antibacterial and antifungal activities. After experimental investigations, 


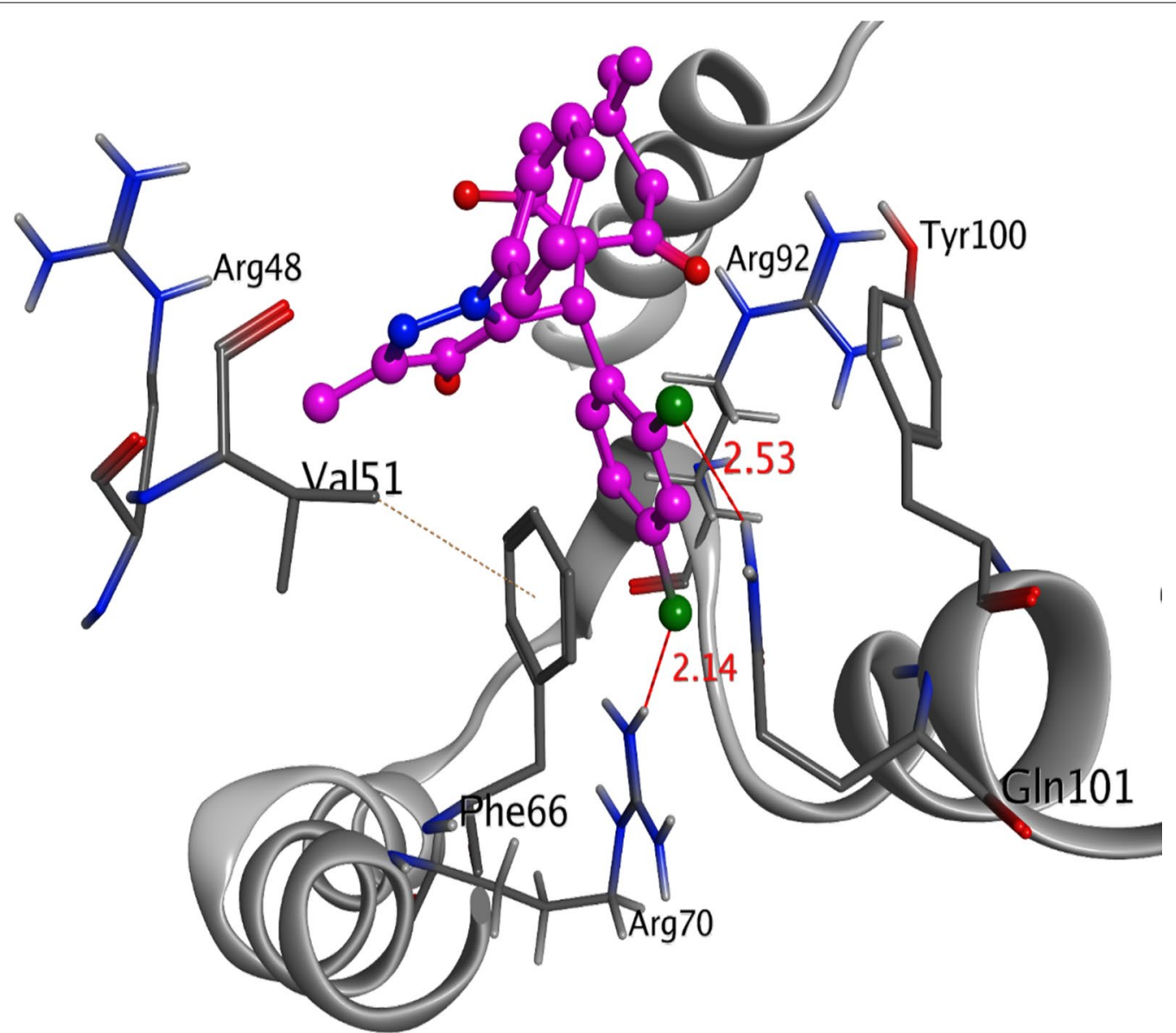

Fig. 2 3-D interaction diagram for the compound $\mathbf{4 l}$ (magenta) presenting a number of electrostatic (red dotted lines) and hydrophobic interactions (orange) with crucial residues of Thymidylate Kinase target protein (gray) from S.aureus

structure-activity relationship profiling was predicted by ligand-based pharmacophore modeling highlighting three features as a requirement for their antimicrobial activity. While Molecular docking predicted the molecular mechanisms of these derivatives with seven different target proteins. Among them, TMK from S. aureus and NMT protein from $C$. albicans were predicted as the most suitable targets for the antibacterial and antifungal activities of these newly synthesized derivatives.

\section{Experimental}

\section{Materials and methods}

General

"All the chemicals were purchased from Aldrich, SigmaAldrich, Fluka etc., and were used without further purification, unless otherwise stated. All melting points were measured on a Gallenkamp melting point apparatus in open glass capillaries and are uncorrected. IR Spectra were measured as $\mathrm{KBr}$ pellets on a Nicolet 6700 FT-IR spectrophotometer. The NMR spectra were recorded on a Varian Mercury Jeol-400 NMR spectrometer. ${ }^{1} \mathrm{H}-\mathrm{NMR}$ $(400 \mathrm{MHz})$, and ${ }^{13} \mathrm{C}-\mathrm{NMR}(100 \mathrm{MHz})$ were run in either deuterated dimethyl sulphoxide (DMSO- $d_{6}$ ) or deuterated chloroform $\left(\mathrm{CDCl}_{3}\right)$. Chemical shifts $(\delta)$ are referred in terms of ppm and $\mathrm{J}$-coupling constants are given in $\mathrm{Hz}$. Mass spectra were recorded on a Jeol of JMS-600 H. Elemental analysis was carried out on Elmer 2400 Elemental Analyzer; CHN mode".

General procedure for Knoevenagel condensation Michael addition for the synthesis of $4 \boldsymbol{a}-\boldsymbol{o}$ (GP1) A mixture of aldehyde 1 (1.5 mmol), 5,5-dimethylcyclohexane-1,3-dione 2 , (1.5 mmol), 3-methyl-1-phenyl-1 $H$-pyrazol-5 $(4 H)$ one $(1.5 \mathrm{mmol})$ and $\mathrm{Et}_{2} \mathrm{NH}(1.5 \mathrm{mmol}, 155 \mu \mathrm{L})$ in $3 \mathrm{~mL}$ of degassed $\mathrm{H}_{2} \mathrm{O}$ was stirred at room temperature for 1-12 h until TLC showed complete disappearance of the reactants. The precipitate was removed by filtration and washed with ether $(3 \times 20 \mathrm{~mL})$. Solid was dried to afford pure products $\mathbf{4 a - 0 .}$ 


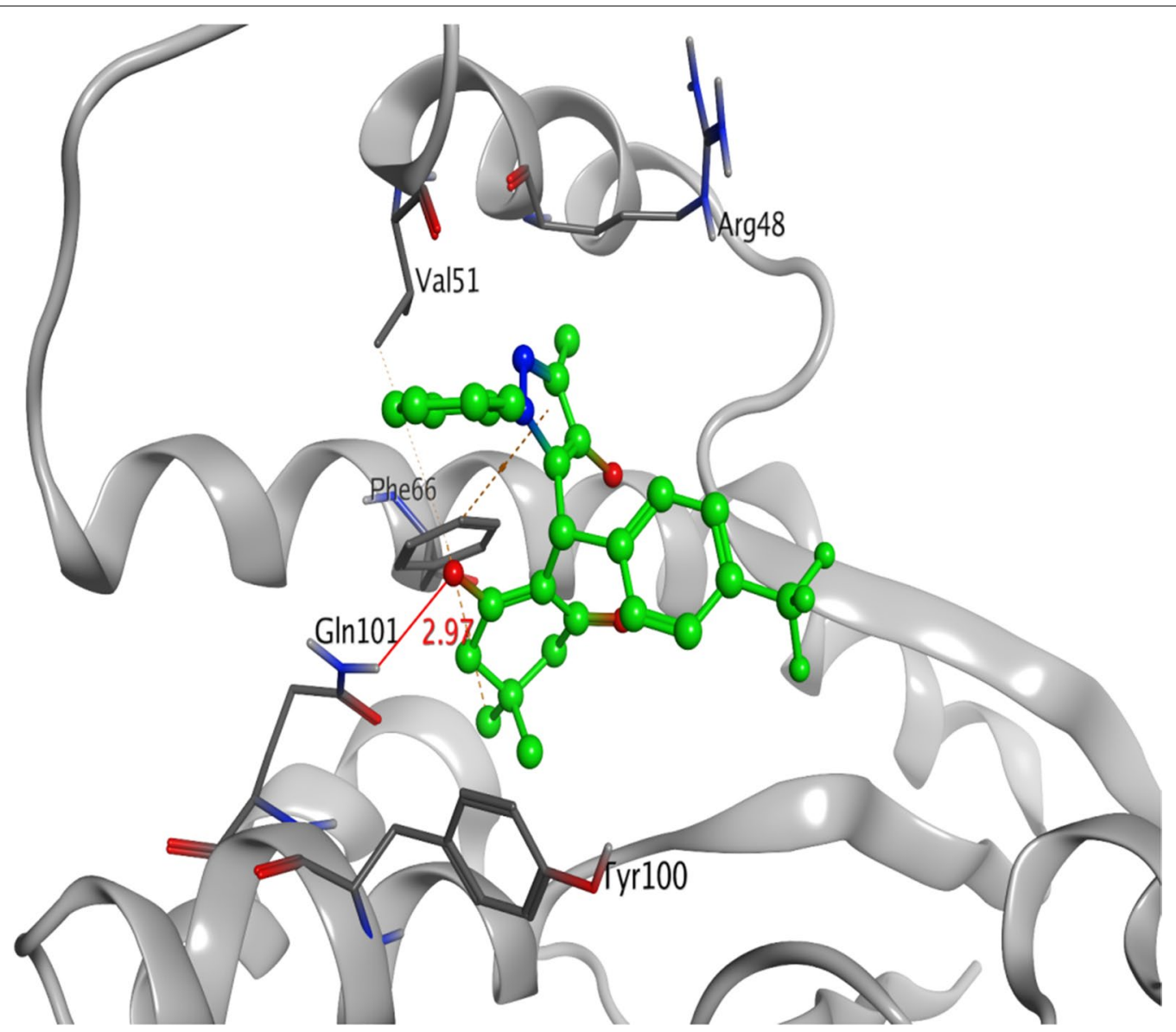

Fig. 3 3D ribbon diagram of the active site of Thymidylate Kinase (grey) from S. aureus species displaying few electrostatic (red line) and multiple hydrophobic and $\pi-\pi$ interactions with hotspot residues (hot pink) responsible for the moderate inhibitory activity of most potent compound $\mathbf{4 k}$

5-((2,4-Dichlorophenyl)(2-hydroxy-4,4-dimethyl-6-oxocyclohex-1-en-1-yl)methyl)-3-methyl-1-phenyl-1H-pyrazol-4-olate diethylaminium salt $4 \boldsymbol{a}$ 4a was prepared according to the general procedure (GP1) from 2,4-dichlorobenzaldehyde yielding orange powdered materials. m.p: $144{ }^{\circ} \mathrm{C}$; IR $\left(\mathrm{CsI}, \mathrm{cm}^{-1}\right): 3451,2984,2868,2719,2492,1598$, $1501,1468,1380,1262 ;{ }^{1} \mathrm{H}-\mathrm{NMR}\left(400 \mathrm{MHz}, \mathrm{DMSO}-d_{6}\right)$ : $8.08(\mathrm{~d}, 1 \mathrm{H}, J=7.3 \mathrm{~Hz}, \mathrm{Ph}), 7.93(\mathrm{~d}, \mathrm{H}, J=7.3 \mathrm{~Hz}, \mathrm{Ph}), 7.42$ $(\mathrm{s}, 1 \mathrm{H}, \mathrm{Ph}), 7.32-7.04(\mathrm{~m}, 5 \mathrm{H}, \mathrm{Ph}), 4.96(\mathrm{~s}, 1 \mathrm{H}, \mathrm{CH}=\mathrm{C})$, $2.85\left(\mathrm{q}, 4 \mathrm{H}, J=7.3 \mathrm{~Hz}, \mathrm{CH}_{2} \mathrm{CH}_{3}\right), 2.12\left(\mathrm{~s}, 3 \mathrm{H}, \mathrm{CH}_{3}\right)$, $1.11\left(\mathrm{t}, 6 \mathrm{H}, J=7.3 \mathrm{~Hz}, \mathrm{CH}_{2} \mathrm{CH}_{3}\right) ;{ }^{13} \mathrm{C}-\mathrm{NMR}(100 \mathrm{MHz}$, DMSO- $\left.d_{6}\right): \delta=157.6,145.5,142.4,140.6,132.1,131.9$, 128.3, 128.0, 126.6, 123.0, 119.1, 100.9, 41.7, 30.9, 13.2, 11.0; LC/MS (ESI): 330.07 [M] ${ }^{+}$for $\mathrm{C}_{18} \mathrm{H}_{16} \mathrm{Cl}_{2} \mathrm{~N}_{2}$; Anal. for $\mathrm{C}_{21} \mathrm{H}_{24} \mathrm{Cl}_{2} \mathrm{~N}_{3} \mathrm{O}$; calcd C, 62.23; H, 5.97; Cl, 17.49; N, 10.37; Found: C, 62.23; H, 5.97; Cl, 17.49; N, 10.37 .

3-Hydroxy-2-((5-hydroxy-3-methyl-1-phenyl-1H-pyrazol-4-yl)(phenyl)methyl)-5,5-dimethylcyclohex-2-enone diethylaminium salt $\mathbf{4} \boldsymbol{b} \mathbf{4 b}$ was prepared according to the general procedure (GP1) from benzaldehyde yielding orange powdered materials. m.p: $102{ }^{\circ} \mathrm{C}$; IR $\left(\mathrm{CsI} \mathrm{cm}^{-1}\right)$ : 3448, 3058, 2957, 2732, 2507, 1582, 1579, 1501, 1492, $1454,1365,1263 ;{ }^{1} \mathrm{H}-\mathrm{NMR}\left(400 \mathrm{MHz}\right.$, DMSO- $\left.d_{6}\right): \delta 15.30$ (s, 1H, OH), 7.92(m, 3H, Ph), 7.33-7.07 (m, 7H, Ph), 5.75 (s, 1H, benzyl-H), $2.86\left(\mathrm{q}, 4 \mathrm{H}, J=7.3 \mathrm{~Hz}, \mathrm{CH}_{2} \mathrm{CH}_{3}\right), 2.16$ (s, $\left.3 \mathrm{H}, \mathrm{CH}_{3}\right), 2.12\left(\mathrm{~s}, 2 \mathrm{H}, \mathrm{CH}_{2}\right), 2.09\left(\mathrm{~s}, 2 \mathrm{H}, \mathrm{CH}_{2}\right), 1.11$ (t, $\left.6 \mathrm{H}, J=7.3 \mathrm{~Hz}, \mathrm{CH}_{2} \mathrm{CH}_{3}\right), 1.10\left(\mathrm{~s}, 3 \mathrm{H}, \mathrm{CH}_{3}\right), 1.00(\mathrm{~s}, 3 \mathrm{H}$, $\left.\mathrm{CH}_{3}\right) ;{ }^{13} \mathrm{C}$-NMR $\left(100 \mathrm{MHz}\right.$, DMSO- $\left.d_{6}\right): \delta=189.8,157.2$, 146.4, 145.8, 145.5, 140.5, 128.4, 128.3, 127.7, 127.2, 119.1, 102.2, 79.2, 41.4, 30.2, 28.8, 12.9, 12.7, 11.00; LC/MS (ESI): 262.1M] $]^{+}$for $\mathrm{C}_{18} \mathrm{H}_{18} \mathrm{~N}_{2}$; Anal. for $\mathrm{C}_{29} \mathrm{H}_{38} \mathrm{~N}_{3} \mathrm{O}_{3}$; calcdC, 73.08; H, 8.04; N, 8.82; Found: C, 73.07; H, 8.05; N, 8.83.

Diethylammonium 5-((4-chlorophenyl)(2-hydroxy-4,4-dimethyl-6-oxocyclohex-1-en-1-yl)methyl)-3-methyl-1-phenyl-1H-pyrazol -4-olate 4c 4c was prepared according to the general procedure (GP1) from 4-chlorobenzaldehyde yielding orange powdered materials. m.p: $92{ }^{\circ} \mathrm{C}$; IR $\left(\mathrm{CsI} \mathrm{cm}{ }^{-1}\right)$ : 3450, 2958, 2868, 2732, 2506, 1702, 1579, 1501, 1487, 1387, 1366, 1318, 1263; ${ }^{1} \mathrm{H}-\mathrm{NMR}(400 \mathrm{MHz}$, DMSO- $\left.d_{6}\right): \delta 15.30(\mathrm{~s}, 1 \mathrm{H}, \mathrm{OH}), 7.34-7.07(\mathrm{~m}, 7 \mathrm{H}, \mathrm{Ph})$, 


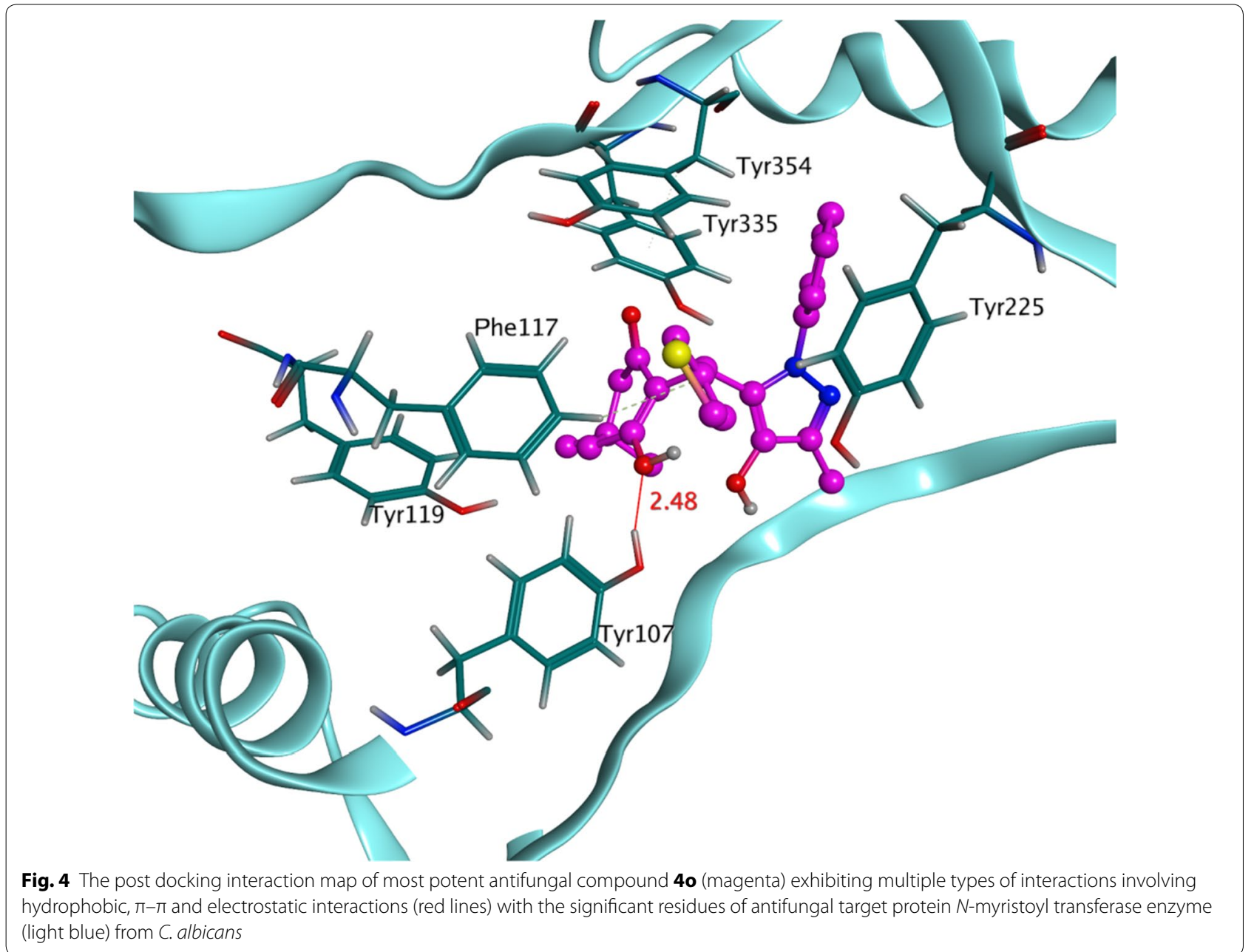

$5.57\left(\mathrm{~s}, 1 \mathrm{H}\right.$, benzyl-H), $2.91\left(\mathrm{q}, 4 \mathrm{H}, J=7.3 \mathrm{~Hz}, \mathrm{CH}_{2} \mathrm{CH}_{3}\right.$ ), 2.19 (s, 3H, $\left.\mathrm{CH}_{3}\right), 2.18\left(\mathrm{~s}, 2 \mathrm{H}, \mathrm{CH}_{2}\right), 2.12\left(\mathrm{~s}, 2 \mathrm{H}, \mathrm{CH}_{2}\right)$, $0.99\left(\mathrm{t}, 6 \mathrm{H}, J=7.3 \mathrm{~Hz}, \mathrm{CH}_{2} \mathrm{CH}_{3}\right), 1.14\left(\mathrm{~s}, 3 \mathrm{H}, \mathrm{CH}_{3}\right), 1.15$ (s, $\left.3 \mathrm{H}, \mathrm{CH}_{3}\right) ;{ }^{13} \mathrm{C}-\mathrm{NMR}\left(100 \mathrm{MHz}\right.$, DMSO- $\left.d_{6}\right): \delta=189.8$, 157.2, 146.4, 145.8, 145.5, 140.5, 128.4, 128.3, 127.7, 127.2, 119.1, 102.2, 79.2, 41.4, 30.2, 28.8, 12.9, 12.7, 11.00; LC/MS (ESI): $262.1 \mathrm{M}]^{+}$for $\mathrm{C}_{18} \mathrm{H}_{17} \mathrm{ClN}_{2}$; Anal. for $\mathrm{C}_{29} \mathrm{H}_{36} \mathrm{ClN}_{3} \mathrm{O}_{3}$; Calcd C, 73.08; H, 8.04; N, 8.82; Found: C, 73.07; H, 8.05; $\mathrm{N}, 8.83, \mathrm{Cl}, 6.21$.

3-Hydroxy-2-((5-hydroxy-3-methyl-1-phenyl-1H-pyrazol-4-yl)(p-tolyl)methyl)-5,5-dimethylcyclohex-2-enone diethylaminium salt $\mathbf{4 d} \mathbf{4 d}$ was prepared according to the general procedure (GP1) from $p$-tolualdehyde yielding orange powdered materials. m.p: $104{ }^{\circ} \mathrm{C}$; IR $\left(\mathrm{CsI}, \mathrm{cm}^{-1}\right)$ : 3450, 3017, 2956, 2732, 2506, 1683, 1581, 1501, 1455, $1386,1318,1260 ;{ }^{1} \mathrm{H}-\mathrm{NMR}\left(400 \mathrm{MHz}, \mathrm{CDCl}_{3}\right): \delta 15.45(\mathrm{~s}$, $1 \mathrm{H}, \mathrm{OH}), 7.67$ (dd, $2 \mathrm{H}, J=7.3 \mathrm{~Hz}, 1.5 \mathrm{~Hz}, \mathrm{Ph}), 7.28$ (dd, $2 \mathrm{H}$, $J=7.3 \mathrm{~Hz}, 1.5 \mathrm{~Hz}, \mathrm{Ph}), 7.20-6.94(\mathrm{~m}, 5 \mathrm{H}, \mathrm{Ph}), 5.62(\mathrm{~s}, 1 \mathrm{H}$, benzyl-H), $2.31\left(\mathrm{~s}, 3 \mathrm{H}, \mathrm{CH}_{3}\right), 2.29\left(\mathrm{~s}, 2 \mathrm{H}, \mathrm{CH}_{2}\right), 2.28(\mathrm{~s}, 3 \mathrm{H}$,
$\left.\mathrm{CH}_{3}\right), 2.23\left(\mathrm{~s}, 2 \mathrm{H}, \mathrm{CH}_{2}\right), 2.18$ (q, 4H, J=7.3 Hz, $\mathrm{CH}_{2} \mathrm{CH}_{3}$ ), $1.01\left(\mathrm{~s}, 6 \mathrm{H}, \mathrm{CH}_{3}\right), 0.84\left(\mathrm{t}, 6 \mathrm{H}, J=7.3 \mathrm{~Hz}, \mathrm{CH}_{2} \mathrm{CH}_{3}\right) ;{ }^{13} \mathrm{C}-$ NMR $\left(100 \mathrm{MHz}, \mathrm{CDCl}_{3}\right): \delta=189.8,168.5,157.9,145.9$, $140.4,128.8,128.7,128.5,127.6,127.3,121.7,121.3,80.3$, 41.7, 31.5, 20.9, 12.6, 11.5; LC/MS (ESI): $276.1[\mathrm{M}]^{+}$for $\mathrm{C}_{19} \mathrm{H}_{20} \mathrm{~N}_{2}$; Anal. for $\mathrm{C}_{30} \mathrm{H}_{40} \mathrm{~N}_{3} \mathrm{O}_{3}$; calcdC, 73.44; $\mathrm{H}, 8.22$; N, 8.56; Found: C, 73.43; H, 8.23; N, 8.57.

3-Hydroxy-2-((5-hydroxy-3-methyl-1-phenyl-1H-pyrazol-4-yl)(m-tolyl)methyl)-5,5-dimethylcyclohex-2-enone diethylaminium salt $4 \boldsymbol{e} \mathbf{4 e}$ was prepared according to the general procedure (GP1) from $m$-tolualdehyde yielding orange powdered materials. m.p: $97^{\circ} \mathrm{C}$; IR $\left(\mathrm{CsI}, \mathrm{cm}^{-1}\right)$ : 3449, 3033, 2956, 2731, 2506, 1581, 1501, 1387, 1318, 1261; ${ }^{1} \mathrm{H}-\mathrm{NMR}\left(400 \mathrm{MHz}, \mathrm{DMSO}-d_{6}\right): \delta 15.45(\mathrm{~s}, 1 \mathrm{H}, \mathrm{OH}), 7.68$ (dd, $2 \mathrm{H}, J=7.3 \mathrm{~Hz}, 1.5 \mathrm{~Hz}, \mathrm{Ph}), 7.63(\mathrm{dd}, 2 \mathrm{H}, J=7.3 \mathrm{~Hz}$, $1.5 \mathrm{~Hz}, \mathrm{Ph}), 7.28-7.06(\mathrm{~m}, 5 \mathrm{H}, \mathrm{Ph}), 5.62(\mathrm{~s}, 1 \mathrm{H}$, benzyl-H), $2.30\left(\mathrm{~s}, 3 \mathrm{H}, \mathrm{CH}_{3}\right), 2.20\left(\mathrm{~s}, 2 \mathrm{H}, \mathrm{CH}_{2}\right), 2.23\left(\mathrm{~s}, 3 \mathrm{H}, \mathrm{CH}_{3}\right)$, $2.18\left(\mathrm{~s}, 2 \mathrm{H}, \mathrm{CH}_{2}\right), 2.25$ (q, $\left.4 \mathrm{H}, J=7.3 \mathrm{~Hz}, \mathrm{CH}_{2} \mathrm{CH}_{3}\right), 1.00$ (s, 6H, $\left.\mathrm{CH}_{3}\right), 0.83\left(\mathrm{t}, 6 \mathrm{H}, J=7.3 \mathrm{~Hz}, \mathrm{CH}_{2} \mathrm{CH}_{3}\right) ;{ }^{13} \mathrm{C}-\mathrm{NMR}$ 
(100 MHz, DMSO- $\left.d_{6}\right): \delta=189.8,168.5,157.9,145.9$, $140.4,128.8,128.7,128.5,127.6,127.3,121.7,121.3,80.3$, 41.7, 31.5, 20.9, 12.6, 11.5; Anal. for $\mathrm{C}_{30} \mathrm{H}_{40} \mathrm{~N}_{3} \mathrm{O}_{3}$; calcdC, 73.44; H, 8.22; N, 8.56; Found: C, 73.43; H, 8.23; N, 8.57.

2-((4-Bromophenyl)(5-hydroxy-3-methyl-1-phenyl-1H-pyrazol-4-yl)methyl)-3-hydroxy-5,5-dimethylcyclohex-2-enone diethylaminium salt $4 \boldsymbol{f}$ 4f was prepared according to the general procedure (GP1) from $p$-bromobenzaldehyde yielding orange powdered materials. m.p: $86^{\circ} \mathrm{C}$; IR $\left(\mathrm{KBr}, \mathrm{cm}^{-1}\right)$ : 3449, 2957, 2868, 2731, 250, 1699, 1579, 1501, 1483, 1388, 1263; ${ }^{1} \mathrm{H}-\mathrm{NMR}(400 \mathrm{MHz}$, DMSO- $\left.d_{6}\right): \delta 15.45(\mathrm{~s}, 1 \mathrm{H}, \mathrm{OH}), 7.91(\mathrm{dd}, 2 \mathrm{H}, J=7.3 \mathrm{~Hz}$, $1.5 \mathrm{~Hz}, \mathrm{Ph}), 7.35-7.26(\mathrm{~m}, 5 \mathrm{H}, \mathrm{Ph}), 7.20-6.96$ (dd, $2 \mathrm{H}$, $\mathrm{J}=7.3 \mathrm{~Hz}, 1.5 \mathrm{~Hz}, \mathrm{Ph}), 5.50(\mathrm{~s}, 1 \mathrm{H}$, benzyl-H), $2.90(\mathrm{q}, 4 \mathrm{H}$, $\left.J=7.3 \mathrm{~Hz}, \mathrm{CH}_{2} \mathrm{CH}_{3}\right), 2.13\left(\mathrm{~s}, 3 \mathrm{H}, \mathrm{CH}_{3}\right), 2.07\left(\mathrm{~s}, 2 \mathrm{H}, \mathrm{CH}_{2}\right)$, $2.05\left(\mathrm{~s}, 2 \mathrm{H}, \mathrm{CH}_{2}\right), 1.14\left(\mathrm{t}, 6 \mathrm{H}, J=7.3 \mathrm{~Hz}, \mathrm{CH}_{2} \mathrm{CH}_{3}\right), 1.12(\mathrm{~s}$, $\left.3 \mathrm{H}, \mathrm{CH}_{3}\right), 0.96\left(\mathrm{~s}, 3 \mathrm{H}, \mathrm{CH}_{3}\right) ;{ }^{13} \mathrm{C}-\mathrm{NMR}(100 \mathrm{MHz}, \mathrm{DMSO}-$ $\left.d_{6}\right): \delta=189.8,157.2,155.9,147.0,145.8,145.5,140.7$, 130.4, 129.6, 129.5, 128.4, 128.2, 122.9, 119.0, 118.8, 101.7, 79.7, 41.4, 31.9, 30.1, 28.3, 12.9, 128, 11.0; LC/MS (ESI): $340.1[\mathrm{M}]^{+}$for $\mathrm{C}_{18} \mathrm{H}_{17} \mathrm{BrN}_{2}$; Anal. for $\mathrm{C}_{29} \mathrm{H}_{37} \mathrm{BrN}_{3} \mathrm{O}_{3}$; calcd C, 62.70; H, 6.71; Br, 14.38; N, 7.56; Found: C, 62.71; $\mathrm{H}, 6.71 ; \mathrm{Br}, 14.39 ; \mathrm{N}, 7.54$.

2-((3-Bromophenyl)(5-hydroxy-3-methyl-1-phenyl-1H-pyrazol-4-yl)methyl)-3-hydroxy-5,5-dimethylcyclohex-2-enone diethylaminium salt $\mathbf{4 g} \mathbf{4 g}$ was prepared according to the general procedure (GP1) from $m$-bromobenzaldehyde yielding rose powdered materials. m.p: $97^{\circ} \mathrm{C}$; IR $\left(\mathrm{KBr}, \mathrm{cm}^{-1}\right)$ : 3447, 2957, 2868, 2730, 2505, 1584, 1501, 1470, 1388, 1365, 1262; ${ }^{1} \mathrm{H}-\mathrm{NMR}(400 \mathrm{MHz}$, DMSO- $\left.d_{6}\right): \delta 15.45(\mathrm{~s}, 1 \mathrm{H}, \mathrm{OH}), 7.92(\mathrm{dd}, 1 \mathrm{H}, J=7.3 \mathrm{~Hz}$, $1.5 \mathrm{~Hz}, \mathrm{Ph}), 7.50(\mathrm{~s}, 1 \mathrm{H}, \mathrm{Ph}), 7.35-7.04(\mathrm{~m}, 8 \mathrm{H}, \mathrm{Ph}), 5.55(\mathrm{~s}$, $1 \mathrm{H}$, benzyl-H), 2.89 (q, $\left.4 \mathrm{H}, J=7.3 \mathrm{~Hz}, \mathrm{CH}_{2} \mathrm{CH}_{3}\right), 2.15$ (s, $\left.3 \mathrm{H}, \mathrm{CH}_{3}\right), 2.09\left(\mathrm{~s}, 2 \mathrm{H}, \mathrm{CH}_{2}\right), 2.06\left(\mathrm{~s}, 2 \mathrm{H}, \mathrm{CH}_{2}\right), 1.14(\mathrm{t}, 6 \mathrm{H}$, $\left.J=7.3 \mathrm{~Hz}, \mathrm{CH}_{2} \mathrm{CH}_{3}\right), 1.10\left(\mathrm{~s}, 3 \mathrm{H}, \mathrm{CH}_{3}\right), 0.98\left(\mathrm{~s}, 3 \mathrm{H}, \mathrm{CH}_{3}\right)$; ${ }^{13} \mathrm{C}-\mathrm{NMR}\left(100 \mathrm{MHz}, \mathrm{DMSO}-d_{6}\right): \delta=189.8,157.2,155.9$, 149.3, 147.0, 145.8, 145.5, 140.7, 140.2, 129.9, 128.4, 128.3, 123.0, 119.0, 118.8, 101.6, 79.1, 41.4, 31.9, 30.1, 28.3, 12.9, 128, 11.0; LC/MS (ESI): $340.1[\mathrm{M}]^{+}$for $\mathrm{C}_{18} \mathrm{H}_{17} \mathrm{BrN}_{2}$; Anal. for $\mathrm{C}_{29} \mathrm{H}_{37} \mathrm{BrN}_{3} \mathrm{O}_{3}$; calcd C, 62.70; $\mathrm{H}, 6.71 ; \mathrm{Br}, 14.38 ; \mathrm{N}$, 7.56; Found: C, 62.71; H, 6.71; Br, 14.39; N, 7.53.

3-Hydroxy-2-((5-hydroxy-3-methyl-1-phenyl-1H-pyrazol-4-yl)(4-nitrophenyl)methyl)-5,5-dimethylcyclohex-2-enone diethylaminium salt $4 \mathbf{h} \mathbf{4 h}$ was prepared according to the general procedure (GP1) from $p$-nitrobenzaldehyde yielding paige powdered materials. m.p: $106{ }^{\circ} \mathrm{C}$; IR $\left(\mathrm{CsI}, \mathrm{cm}^{-1}\right)$ : 3451, 2958, 2869, 2732, 2503, 1707, 1597, 1513, 1387, 1320, 1267; ${ }^{1} \mathrm{H}-\mathrm{NMR}(400 \mathrm{MHz}$, $\left.\mathrm{CDCl}_{3}\right): \delta 15.40(\mathrm{~s}, 1 \mathrm{H}, \mathrm{OH}), 8.02(\mathrm{dd}, 2 \mathrm{H}, J=7.3 \mathrm{~Hz}$, $1.5 \mathrm{~Hz}, \mathrm{Ph}), 7.61$ (dd, $2 \mathrm{H}, J=7.3 \mathrm{~Hz}, 1.5 \mathrm{~Hz}, \mathrm{Ph}), 7.31-7.19$ (m, 5H, Ph), $5.72(\mathrm{~s}, 1 \mathrm{H}$, benzyl-H), $2.70(\mathrm{q}, 4 \mathrm{H}, J=7.3 \mathrm{~Hz}$, $\left.\mathrm{CH}_{2} \mathrm{CH}_{3}\right), 2.27\left(\mathrm{~s}, 3 \mathrm{H}, \mathrm{CH}_{3}\right), 2.24\left(\mathrm{~s}, 2 \mathrm{H}, \mathrm{CH}_{2}\right), 2.19(\mathrm{~s}, 2 \mathrm{H}$, $\left.\mathrm{CH}_{2}\right), 1.07\left(\mathrm{~s}, 6 \mathrm{H}, \mathrm{CH}_{3}\right), 1.02\left(\mathrm{t}, 6 \mathrm{H}, J=7.3 \mathrm{~Hz}, \mathrm{CH}_{2} \mathrm{CH}_{3}\right)$; ${ }^{13} \mathrm{C}-\mathrm{NMR}\left(100 \mathrm{MHz}, \mathrm{CDCl}_{3}\right): \delta=189.8,157.9,145.9$, 140.4, 128.7, 128.6, 128.2, 127.9, 127.7, 125.3, 124.8, 121.6, 121.2, 80.3, 42.3, 31.6, 21.7, 11.4; LC/MS (ESI): $307.1[\mathrm{M}]^{+}$ for $\mathrm{C}_{18} \mathrm{H}_{17} \mathrm{~N}_{3} \mathrm{O}_{2}$; Anal. for $\mathrm{C}_{29} \mathrm{H}_{37} \mathrm{~N}_{4} \mathrm{O}_{5}$; calcd C, 66.77; $\mathrm{H}$, 7.15; N, 10.74; Found: C, 66.75; H, 7.16; N, 10.75 .

3-Hydroxy-2-((5-hydroxy-3-methyl-1-phenyl-1H-pyrazol-4-yl)(3-nitrophenyl)methyl)-5,5-dimethylcyclohex2-enone diethylaminium salt $4 \mathbf{i} \mathbf{4 i}$ was prepared according to the general procedure (GP1) from $m$-nitrobenzaldehyde yielding white paige powdered materials. m.p: $99^{\circ} \mathrm{C}$; IR (CsI, cm ${ }^{-1}$ ): 3447, 3067, 2958, 2731, 2560, 1705, 1597, 1502, 1387, 1348, 1265; ${ }^{1} \mathrm{H}-\mathrm{NMR}$ $\left(400 \mathrm{MHz}, \mathrm{CDCl}_{3}\right): \delta 15.30(\mathrm{~s}, 1 \mathrm{H}, \mathrm{OH}), 8.02(\mathrm{dd}, 2 \mathrm{H}$, $J=7.3 \mathrm{~Hz}, 1.5 \mathrm{~Hz}, \mathrm{Ph}), 7.61(\mathrm{dd}, 2 \mathrm{H}, J=7.3 \mathrm{~Hz}, 1.5 \mathrm{~Hz}$, $\mathrm{Ph}), 7.31-7.19(\mathrm{~m}, 5 \mathrm{H}, \mathrm{Ph}), 5.72(\mathrm{~s}, 1 \mathrm{H}$, benzyl-H), 2.64 (q, $\left.4 \mathrm{H}, J=7.3 \mathrm{~Hz}, \mathrm{CH}_{2} \mathrm{CH}_{3}\right), 2.27\left(\mathrm{~s}, 3 \mathrm{H}, \mathrm{CH}_{3}\right), 2.25$ (s, $\left.2 \mathrm{H}, \mathrm{CH}_{2}\right), 2.18\left(\mathrm{~s}, 2 \mathrm{H}, \mathrm{CH}_{2}\right), 1.05\left(\mathrm{~s}, 6 \mathrm{H}, \mathrm{CH}_{3}\right), 1.02(\mathrm{t}$, $\left.6 \mathrm{H}, J=7.3 \mathrm{~Hz}, \mathrm{CH}_{2} \mathrm{CH}_{3}\right) ;{ }^{13} \mathrm{C}-\mathrm{NMR}\left(100 \mathrm{MHz}, \mathrm{CDCl}_{3}\right)$ : $\delta=189.8,157.9,145.9,140.4,128.7,128.6,128.2,127.9$, 127.7, 125.3, 124.8, 121.6, 121.2, 80.3, 42.3, 31.6, 21.7, 11.6; LC/MS (ESI): $307.1[\mathrm{M}]^{+}$for $\mathrm{C}_{18} \mathrm{H}_{17} \mathrm{~N}_{3} \mathrm{O}_{2}$; Anal. for $\mathrm{C}_{29} \mathrm{H}_{37} \mathrm{~N}_{4} \mathrm{O}_{5}$; calcd C, 66.77; H, 7.15; N, 10.74; Found: C, 66.75; H, 7.16; N, 10.75 .

3-Hydroxy-2-((5-hydroxy-3-methyl-1-phenyl-1H-pyrazol-4-yl)(4-methoxyphenyl)methyl)-5,5-dimethylcyclo hex-2-enone diethylaminium salt $4 \mathbf{j} \mathbf{4} \mathbf{j}$ was prepared according to the general procedure (GP1) from anisaldehyde yielding deep orange materials. m.p: $84{ }^{\circ} \mathrm{C}$; IR (CsI, $\left.\mathrm{cm}^{-1}\right)$ : 3451, 2956, 2835, 2732, 2507, 1681, 1598, 1502, 1456, 1366, 1318, $1261 ;{ }^{1} \mathrm{H}-\mathrm{NMR}\left(400 \mathrm{MHz}, \mathrm{CDCl}_{3}\right): \delta$ $15.35(\mathrm{~s}, 1 \mathrm{H}, \mathrm{OH}), 7.64$ (dd, $2 \mathrm{H}, J=7.3 \mathrm{~Hz}, 1.5 \mathrm{~Hz}, \mathrm{Ph})$, $7.27(\mathrm{dd}, 2 \mathrm{H}, J=7.3 \mathrm{~Hz}, 1.5 \mathrm{~Hz}, \mathrm{Ph}), 7.14-6,68(\mathrm{~m}, 5 \mathrm{H}, \mathrm{Ph})$, $5.59\left(\mathrm{~s}, 1 \mathrm{H}\right.$, benzyl-H), $3.69\left(\mathrm{~s}, 3 \mathrm{H}, \mathrm{OCH}_{3}\right), 2.33(\mathrm{q}, 4 \mathrm{H}$, $\left.J=7.3 \mathrm{~Hz}, \mathrm{CH}_{2} \mathrm{CH}_{3}\right), 2.27\left(\mathrm{~s}, 3 \mathrm{H}, \mathrm{CH}_{3}\right), 2.25\left(\mathrm{~s}, 2 \mathrm{H}, \mathrm{CH}_{2}\right)$, $2.17\left(\mathrm{~s}, 2 \mathrm{H}, \mathrm{CH}_{2}\right), 0.99\left(\mathrm{~s}, 6 \mathrm{H}, \mathrm{CH}_{3}\right), 0.83(\mathrm{t}, 6 \mathrm{H}, J=7.3 \mathrm{~Hz}$, $\left.\mathrm{CH}_{2} \mathrm{CH}_{3}\right) ;{ }^{13} \mathrm{C}$-NMR $\left(100 \mathrm{MHz}, \mathrm{CDCl}_{3}\right): \delta=189.8,157.9$, 145.9, 140.4, 136.8, 128.8, 128.6, 125.4, 121.7, 121.3, 114.4, 113.4, 113.2, 80.3, 55.4, 41.7, 31.4, 11.2; LC/MS (ESI): $292.1[\mathrm{M}]^{+}$for $\mathrm{C}_{19} \mathrm{H}_{20} \mathrm{~N}_{2} \mathrm{O}$; Anal. for $\mathrm{C}_{30} \mathrm{H}_{40} \mathrm{~N}_{3} \mathrm{O}_{4}$; calcd C, 71.12; H, 7.96; N, 8.29; Found: C, 71.11; H, 7.97; N, 8.31.

2-((4-Fluorophenyl)(5-hydroxy-3-methyl-1-phenyl-1H-pyrazol-4-yl)methyl)-3-hydroxy-5,5-dimethylcyclohex-2-enone diethylaminium salt $\mathbf{4 k} \mathbf{4} \mathbf{k}$ was prepared according to the general procedure (GP1) from $p$-fluorobenzaldehyde yielding orange powdered materials. m.p: $99^{\circ} \mathrm{C}$; $\mathrm{IR}\left(\mathrm{KBr}, \mathrm{cm}^{-1}\right)$ : 3450, 3.35, 2958, 2869, 2731, 2507, 1598, 1580, 1501，1387，1262; ${ }^{1} \mathrm{H}-\mathrm{NMR}$ 
(400 MHz, DMSO- $\left.d_{6}\right): \delta 15.45(\mathrm{~s}, 1 \mathrm{H}, \mathrm{OH}), 7.89-7.83(\mathrm{dd}$, $2 \mathrm{H}, J=7.3 \mathrm{~Hz}, 1.5 \mathrm{~Hz}, \mathrm{Ph}), 7.32-7.28(\mathrm{dd}, 2 \mathrm{H}, J=7.3 \mathrm{~Hz}$, $1.5 \mathrm{~Hz}, \mathrm{Ph}), 7.20-6.94(\mathrm{~m}, 5 \mathrm{H}, \mathrm{Ph}), 5.53(\mathrm{~s}, 1 \mathrm{H}$, benzyl$\mathrm{H}), 2.90\left(\mathrm{q}, 4 \mathrm{H}, J=7.3 \mathrm{~Hz}, \mathrm{CH}_{2} \mathrm{CH}_{3}\right), 2.16\left(\mathrm{~s}, 3 \mathrm{H}, \mathrm{CH}_{3}\right)$, $2.11\left(\mathrm{~s}, 2 \mathrm{H}, \mathrm{CH}_{2}\right), 2.07\left(\mathrm{~s}, 2 \mathrm{H}, \mathrm{CH}_{2}\right), 1.14(\mathrm{t}, 6 \mathrm{H}, J=7.3 \mathrm{~Hz}$, $\left.\mathrm{CH}_{2} \mathrm{CH}_{3}\right), 1.11\left(\mathrm{~s}, 3 \mathrm{H}, \mathrm{CH}_{3}\right), 0.97\left(\mathrm{~s}, 3 \mathrm{H}, \mathrm{CH}_{3}\right) ;{ }^{13} \mathrm{C}-\mathrm{NMR}$ $\left(100 \mathrm{MHz}, \mathrm{DMSO}-d_{6}\right): \delta=189.8,157.2,147.0,145.7$, 140.2, 128.6, 128.5, 128.3, 123.3, 119.2, 118.9, 113.6, 102.4, 102.3, 79.2, 41.4, 31.3, 30.1, 28.7, 12.8, 12.6, 11.0; LC/MS (ESI): $280.1[\mathrm{M}]^{+}$For $\mathrm{C}_{18} \mathrm{H}_{17} \mathrm{FN}_{2}$; Anal. for $\mathrm{C}_{29} \mathrm{H}_{37} \mathrm{FN}_{3} \mathrm{O}_{3}$; calcd C, 70.42; H, 7.54; F, 3.84; N, 8.50; Found: C, 70.43; H, 7.54; F, 3.83; N, 8.49.

3-Hydroxy-2-((5-hydroxy-3-methyl-1-phenyl-1H-pyrazol-4-yl)(4-(trifluoromethyl)phenyl)methyl)-5, 5-dimethylcyclohex-2-enone diethylaminium salt $4 \boldsymbol{l}$ 4l was prepared according to the general procedure (GP1) from $p$-trifluoromethylbenzaldehyde yielding yellow powdered materials. m.p: $96{ }^{\circ} \mathrm{C}$; IR $\left(\mathrm{CsI}, \mathrm{cm}^{-1}\right): 3451,2959,2870$, 2733, 2506, 1615, 1598, 1502, 1387, 1325, 1266; ${ }^{1} \mathrm{H}-\mathrm{NMR}$ $\left(400 \mathrm{MHz}, \mathrm{DMSO}-d_{6}\right): \delta 16.45(\mathrm{~s}, 1 \mathrm{H}, \mathrm{OH}), 7.94-7.90(\mathrm{dd}$, $2 \mathrm{H}, J=7.3 \mathrm{~Hz}, 1.5 \mathrm{~Hz}, \mathrm{Ph}), 7.57-7.44(\mathrm{dd}, 2 \mathrm{H}, J=7.3 \mathrm{~Hz}$, $1.5 \mathrm{~Hz}, \mathrm{Ph}), 7.34-7.06(\mathrm{~m}, 5 \mathrm{H}, \mathrm{Ph}), 5.76(\mathrm{~s}, 1 \mathrm{H}$, benzyl$\mathrm{H}), 2.91\left(\mathrm{q}, 4 \mathrm{H}, J=7.3 \mathrm{~Hz}, \mathrm{CH}_{2} \mathrm{CH}_{3}\right), 2.19\left(\mathrm{~s}, 3 \mathrm{H}, \mathrm{CH}_{3}\right)$, $2.12\left(\mathrm{~s}, 2 \mathrm{H}, \mathrm{CH}_{2}\right), 2.10\left(\mathrm{~s}, 2 \mathrm{H}, \mathrm{CH}_{2}\right), 1.15(\mathrm{t}, 6 \mathrm{H}, J=7.3 \mathrm{~Hz}$, $\left.\mathrm{CH}_{2} \mathrm{CH}_{3}\right), 1.11\left(\mathrm{~s}, 3 \mathrm{H}, \mathrm{CH}_{3}\right), 1.00\left(\mathrm{~s}, 3 \mathrm{H}, \mathrm{CH}_{3}\right) ;{ }^{13} \mathrm{C}-\mathrm{NMR}$ $\left(100 \mathrm{MHz}, \mathrm{DMSO}-d_{6}\right): \delta=157.2,147.0,145.7,140.2$, 128.6, 128.5, 128.3, 123.3, 119.2, 118.9, 113.6, 102.4, 102.3, 79.2, 41.4, 31.3, 30.1, 28.7, 12.8, 12.6, 11.0; LC/MS (ESI): $330.13[\mathrm{M}]^{+}$for $\mathrm{C}_{19} \mathrm{H}_{17} \mathrm{~F}_{3} \mathrm{~N}_{2}$; Anal. for $\mathrm{C}_{30} \mathrm{H}_{37} \mathrm{~F}_{3} \mathrm{~N}_{3} \mathrm{O}_{3}$; calcd C, 66.16; H, 6.85; F, 10.46; N, 7.72; Found: C, 66.17; H, 6.86; F, 10.45; N, 7.71 .

5-((2,6-Dichlorophenyl)(2-hydroxy-4,4-dimethyl-6-oxocyclohex-1-en-1-yl)methyl)-3-methyl-1-phenyl-1H-py razol-4-olate diethylaminium salt $\mathbf{4 m} \mathbf{4 m}$ was prepared according to the general procedure (GP1) from 2,6-dicholorobenzaldehyde yielding deep orange powdered materials. m.p: $142{ }^{\circ} \mathrm{C}$; IR $\left(\mathrm{CsI}, \mathrm{cm}^{-1}\right): 3459,3117$, 3061, 2973, 2834, 2479, 1657, 1646, 1596, 1500, 1431, 1311, 153; ${ }^{1} \mathrm{H}-\mathrm{NMR}$ (400 MHz, DMSO-d6): $8.08(\mathrm{~d}, 1 \mathrm{H}$, $J=7.3 \mathrm{~Hz}, \mathrm{Ph}), 7.93(\mathrm{~d}, \mathrm{H}, J=7.3 \mathrm{~Hz}, \mathrm{Ph}), 7.42(\mathrm{~s}, 1 \mathrm{H}$, $\mathrm{Ph}), 7.32-7.04(\mathrm{~m}, 5 \mathrm{H}, \mathrm{Ph}), 4.96(\mathrm{~s}, 1 \mathrm{H}, \mathrm{CH}=\mathrm{C}), 2.85(\mathrm{q}$, $\left.4 \mathrm{H}, J=7.3 \mathrm{~Hz}, \mathrm{CH}_{2} \mathrm{CH}_{3}\right), 2.12\left(\mathrm{~s}, 3 \mathrm{H}, \mathrm{CH}_{3}\right), 1.11(\mathrm{t}, 6 \mathrm{H}$, $\left.J=7.3 \mathrm{~Hz}, \mathrm{CH}_{2} \mathrm{CH}_{3}\right) ;{ }^{13} \mathrm{C}-\mathrm{NMR}\left(100 \mathrm{MHz}, \mathrm{DMSO}-d_{6}\right)$ : $\delta=161.6,160.1,150.0,148.0,132.9,132.7,131.3,129.0$, 128.9, 128.5, 128.1, 118.1, 117.8, 14.4; LC/MS (ESI): 330.07 $[\mathrm{M}]^{+}$for $\mathrm{C}_{18} \mathrm{H}_{16} \mathrm{Cl}_{2} \mathrm{~N}_{2}$; Anal. for $\mathrm{C}_{17} \mathrm{H}_{12} \mathrm{Cl}_{2} \mathrm{~N}_{2} \mathrm{O}$; calcd C, $61.65 ; \mathrm{H}, 3.65 ; \mathrm{Cl}, 21.41 ; \mathrm{N}, 8.46$; Found: $\mathrm{C}, 61.64 ; \mathrm{H}, 3.63$; Cl, 21.40; N, 8.44.

5-((2-Hydroxy-4,4-dimethyl-6-oxocyclohex-1-en-1-yl) (naphthalen-2-yl)methyl)-3-methyl-1-phenyl-1H-pyraz ol-4-olate diethylaminium salt $4 \mathbf{n}$ 4n was prepared according to the general procedure (GP1) from naphthaldehyde yielding orange powdered materials. m.p: $102{ }^{\circ} \mathrm{C}$; IR (CsI, cm $\left.{ }^{-1}\right)$ : 3452, 3053, 2956, 2729, 2500, 1692, 1579, 1502, 1387, 1320, 1268; ${ }^{1} \mathrm{H}-\mathrm{NMR}\left(400 \mathrm{MHz}, \mathrm{DMSO}-d_{6}\right)$ : $15.32(\mathrm{~s}, 1 \mathrm{H}, \mathrm{OH}), 7.96-7.26(\mathrm{~m}, 8 \mathrm{H}, \mathrm{Ph}), 5.75(\mathrm{~s}, 1 \mathrm{H}$, benzyl-H), $2.27\left(\mathrm{q}, 4 \mathrm{H}, J=7.3 \mathrm{~Hz}, \mathrm{CH}_{2} \mathrm{CH}_{3}\right), 2.20\left(\mathrm{~s}, 3 \mathrm{H}, \mathrm{CH}_{3}\right)$, $2.01\left(\mathrm{~s}, 2 \mathrm{H}, \mathrm{CH}_{2}\right), 2.00\left(\mathrm{~s}, 2 \mathrm{H}, \mathrm{CH}_{2}\right), 1.06\left(\mathrm{~s}, 6 \mathrm{H}, \mathrm{CH}_{3}\right), 0.64$ $\left(\mathrm{t}, 6 \mathrm{H}, J=7.3 \mathrm{~Hz}, \mathrm{CH}_{2} \mathrm{CH}_{3}\right){ }^{13} \mathrm{C}-\mathrm{NMR}(100 \mathrm{MHz}$, DMSO$\left.d_{6}\right): \delta=192.3,156.1,146.7,139.3,128.7,128.7,126,121.7$, 121.30, 103.6, 78.8, 42.1, 31.3, 12.6; LC/MS (ESI): 312.0 $[\mathrm{M}]^{+}$for $\mathrm{C}_{22} \mathrm{H}_{20} \mathrm{~N}_{2}$; Anal. for $\mathrm{C}_{27} \mathrm{H}_{36} \mathrm{~N}_{3} \mathrm{O}_{3} \mathrm{~S}$; calcd C, 67.19; H, 7.52; N, 8.71; S, 6.64; Found: C, 67.20; H, 7.52; N, 8.73.

3-Hydroxy-2-((5-hydroxy-3-methyl-1-phenyl-1H-pyrazol-4-yl)(thiophen-2-yl)methyl)-5,5-dimethylcyclohex2-enone diethylaminium salt 40 4o was prepared according to the general procedure (GP1) from thiophenaldehyde yielding brown powdered materials. m.p: $87^{\circ} \mathrm{C}$; IR $\left(\mathrm{KBr}, \mathrm{cm}^{-1}\right): 3450,3063,2956,2731,2505,1681,1580$, 1501, 1387, 1366, 1261; ${ }^{1} \mathrm{H}-\mathrm{NMR}\left(400 \mathrm{MHz}, \mathrm{CDCl}_{3}\right): \delta$ $15.32(\mathrm{~s}, 1 \mathrm{H}, \mathrm{OH}), 7.71-6.64(\mathrm{~m}, 8 \mathrm{H}, \mathrm{Ph}), 5.81(\mathrm{~s}, 1 \mathrm{H}$, benzyl-H), $2.47\left(\mathrm{q}, 4 \mathrm{H}, \mathrm{J}=7.3 \mathrm{~Hz}, \mathrm{CH}_{2} \mathrm{CH}_{3}\right.$ ), 2.36 (s, 3H, CH3), 2.27(s, 2H, $\left.\mathrm{CH}_{2}\right), 2.23\left(\mathrm{~s}, 2 \mathrm{H}, \mathrm{CH}_{2}\right), 1.12\left(\mathrm{~s}, 6 \mathrm{H}, \mathrm{CH}_{3}\right)$, $0.98\left(\mathrm{t}, 6 \mathrm{H}, J=7.3 \mathrm{~Hz}, \mathrm{CH}_{2} \mathrm{CH}_{3}\right) ;{ }^{13} \mathrm{C}-\mathrm{NMR}(100 \mathrm{MHz}$, $\left.\mathrm{CDCl}_{3}\right): \delta=192.3,156.1,146.7,139.3,128.7,128.7,126$, 121.7, 121.30, 103.6, 78.8, 42.1, 31.3, 12.6; LC/MS (ESI): $268.1[\mathrm{M}]^{+}$for: $\mathrm{C}_{16} \mathrm{H}_{16} \mathrm{~N}_{2} \mathrm{~S}$; Anal. for $\mathrm{C}_{27} \mathrm{H}_{36} \mathrm{~N}_{3} \mathrm{O}_{3} \mathrm{~S}$; calcd C, 67.19; H, 7.52; N, 8.71; S, 6.64; Found: C, 67.20; H, 7.52; $\mathrm{N}, 8.73 ; \mathrm{S}, 6.65$.

\section{Antibacterial activity studies}

The antimicrobial studies were carried out according to reported methodology in the following literature reported by Barakat et al. [12, 13, 23] including initial screening and determination of MIC.

\section{In-silico predictions \\ Pharmacophore modeling}

A ligand-based pharmacophore model was developed by using MOE 2017 [24] suite. Where, a training set representing the most active lead analogs $[12,13]$ was selected, energy minimized and submitted to flexible alignment for analyzing the shared spatial arrangement of their pharmacophoric features. Generated hypotheses were ranked based on their accuracy scoring and atomic overlap. Among the highest ranked hypotheses, the best pharmacophore showing $100 \%$ accuracy was selected. This selected model was validated for its predictive efficacy by overlapping representative active analogs over it and calculating the RMSD (root mean square distance) between the query and mapped compounds. 


\section{Docking simulation}

To predict the most suitable targets and inhibition mechanisms for the antibacterial and antifungal activities of the newly synthesized pyrazole-dimedone derivatives, reference compounds i.e. ciprofloxacin and fluconazole were submitted in Binding DB [25]. Binding DB works on the principle that similar compounds tend to have the same target proteins and seven proteins were chosen; four proteins i.e. Dihydrofolate Reductase (PDB ID 3FYV), Gyrase B (PDB ID 4URM), Thymidylate Kinase (TMK) (PDB ID 4QGG) and Sortase A (PDB ID 2MLM) from S. aureus for antibacterial (ciprofloxacin) and three proteins (Dihydrofolate Reductase (DHFR) (PDB ID 4HOF), Secreted Aspartic Protease (PDB ID 3Q70), and $N$-myristoyl transferase (PDB ID 1IYL) from C. Albicans for antifungal (fluconazole) compounds. The crystal structures of the seven target proteins were fetched from Protein Data Bank (www.rcsb.org/pdb) and all the proteins were prepared, charged, protonated and minimized via MOE 2016 suite. The chemical structures of synthesized compounds were built and saved in their 3D conformations by Builder tool incorporated in MOE 2016. Further protonation, minimization, charge application and atom-type corrections were also done by MOE 2016. Before docking, the efficiency of docking software was validated via redocking the crystallized ligand back into the pocket of significant antibacterial and antifungal target proteins. After redocking experiment (Additional file 1: Figures S1 and S2), we found MOE as the appropriate software to continue our in silico work with this software.

\section{Additional file}

Additional file 1. Additional information.

\section{Authors' contributions}

AB conceived and designed the experiments; BMA-Q and MA performed the experiments; $A M A$ analyzed the data; $A B$ contributed reagents/materials/analysis tools; MHA carried out the antimicrobial activity; MT, SN, and ZU-H carried out pharmacophore modeling and molecular docking studies; $A B$ wrote the paper. All authors read and approved the final manuscript.

\begin{abstract}
Author details
${ }^{1}$ Department of Chemistry, Faculty of Science, King Saud University, P. O. Box 2455, Riyadh 11451, Saudi Arabia. ${ }^{2}$ Department of Chemistry, Faculty of Science, Alexandria University, P. O. Box 426, Ibrahimia, 21321 Alexandria, Egypt. ${ }^{3}$ Department of Pharmaceutical Chemistry, Faculty of Pharmacy, Alexandria University, Alexandria 21521, Egypt. ${ }^{4}$ Microbiology and Immunology Department, Faculty of Pharmacy, Al-Azhar University, Cairo, Egypt. ${ }^{5}$ Division of Microbiology, Pharmaceutics Department, College of Pharmacy, King Saud University, P. O. Box 2457, Riyadh 11451, Saudi Arabia. ${ }^{6}$ Dr. Panjwani Center for Molecular Medicine and Drug Research, International Center for Chemical and Biological Sciences, University of Karachi, Karachi 75210, Pakistan.
\end{abstract}

\section{Acknowledgements}

The authors would like to extend their sincere appreciation to the Deanship of Scientific Research at King Saud University for its funding this Research group NO (RGP-257).

\section{Competing interests}

The authors declare that they have no competing interests.

Ethics approval and consent to participate

Not applicable.

\section{Publisher's Note}

Springer Nature remains neutral with regard to jurisdictional claims in published maps and institutional affiliations.

Received: 3 January 2018 Accepted: 7 March 2018

Published online: 14 March 2018

\section{References}

1. WHO. The burden of health care-associated infection worldwide. 2016. http://www.who.int/gpsc/country_work/burden_hcai/en/Accessed 10 Aug 2016

2. Khan MF, Alam MM, Verma G, Akhtar W, Akhter M, Shaquiquzzaman M (2016) The therapeutic voyage of pyrazole and its analogs: a review. Eur J Med Chem 14(120):170-201

3. Sullivan TJ, Truglio JJ, Boyne ME, Novichenok P, Zhang X, Stratton CF, Li H-J, Kaur T, Amin A, Johnson F, Slayden RA, Kisker C, Tonge PJ (2006) High affinity InhA inhibitors with activity against drug-resistant strains of Mycobacterium tuberculosis. ACS Chem Biol 6:43-53

4. Gilbert AM, Failli A, Shumsky J, Yang Y, Severin A, Singh G, Hu W, Keeney D, Petersen PJ, Katz AH (2006) Pyrazolidine-3, 5-diones and 5-hydroxy-1 H-pyrazol-3 (2 H)-ones, inhibitors of UDP-N-acetylenolpyruvyl glucosamine reductase. J Med Chem 49:6027-6063

5. Isloor AM, Kalluraya B, Shetty P (2009) Regioselective reaction: synthesis, characterization and pharmacological studies of some new Mannich bases derived from 1,2,4-triazoles. Eur J Med Chem 44:3784-3787

6. Magedov IV, Manpadi M, Slambrouck SV, Steelant WF, Rozhkova E, Przheval'skii NM, Rogelj S, Kornienko A (2007) Discovery and investigation of antiproliferative and apoptosis-inducing properties of new heterocyclic podophyllotoxin analogues accessible by a one-step multicomponent synthesis. J Med Chem 50:5183-5192

7. Szab ó G, Fischer J, Kis-Varga Á, Gyires K (2007) New celecoxib derivatives as anti-inflammatory agents. J Med Chem 51:142-147

8. Prasad YP, Rao AL, Prasoona L, Murali K, Kumar PR (2005) Synthesis and antidepressant activity of some 1,3,5-triphenyl-2-pyrazolines and 3-(2"-hydroxy naphthalen-1"-yl)-1, 5-diphenyl-2-pyrazolines. Bioorg Med Chem Lett 15:5030-5034

9. Ozdemir Z, Kandilci HB, Gumusel B, Calis U, Bilgin AA (2007) Synthesis and studies on antidepressant and anticonvulsant activities of some 3-(2-furyl)-pyrazoline derivatives. Eur J Med Chem 42:373-379

10. Şener A, KasimŞener M, Bildmci I, Kasimogullari R, Akçamur Y (2002) Studies on the reactions of cyclic oxalyl compounds with hydrazines or hydrazones: synthesis and reactions of 4-benzoyl-1-(3-nitrophenyl)5-phenyl-1H-pyrazole-3-carboxylic acid. J Heterocycl Chem 39:869-875

11. Wachter GA, Hartmann RW, Sergejew T, Grun GL, Ledergerber D (1996) Tetrahydronaphthalenes: influence of heterocyclic substituents on inhibition of steroidogenic enzymes P450 arom and P450 17. J Med Chem 39:834-841

12. Elshaier YAMM, Barakat A, Al-Qahtany BM, Al-Majid AM, Al-Agamy MH (2016) Synthesis of pyrazole-thiobarbituric acid derivatives: antimicrobial activity and docking studies. Molecules 21:1337-1354

13. Barakat A, Al-Qahtani BM, Al-Majid AM, Ali M, Mabkhot YN, Al-Agamy MHM, Wadood A (2016) Synthesis, characterization, anti-microbial activity and molecular docking studies of combined pyrazol-barbituric acid pharmacophore. Trop J Pharma Res 15:1319-1326

14. Kaya M, Demir E, Bekci H (2013) Synthesis, characterization and antimicrobial activity of novel xanthene sulfonamide and carboxamide derivatives. J Enzym Inhib Med Chem 28(5):885-893

15. Kaya M, Basar E, Colak F (2011) Synthesis and antimicrobial activity of some bisoctahydroxanthene-1, 8-dione derivatives. Med Chem Res 20(8):1214-1219 
16. Sangani CB, Shah NM, Patel MP, Patel RG (2012) Microwave assisted synthesis of novel $4 \mathrm{H}$-chromene derivatives bearing phenoxypyrazole and their antimicrobial activity assess. J Serb Chem Soc 77(9):1 165-1174

17. Singh DP, Kumar R, Surain P, Aneja KR (2014) Spectroscopic and antimicrobial studies of macrocyclic metal complexes derived from 1,8-diaminonaphthalene and dimedone. J Incl Phenom Macro 78(1-4):363-369

18. Wang D, Gao F (2013) Quinazoline derivatives: synthesis and bioactivities. Chem Cent J 7(1):95

19. Shahi M, Foroughifar N, Mobinikhaledi A (2015) Synthesis and antimicrobial activity of some tetrahydro quinolone diones and pyrano [2, 3-d] pyrimidine derivatives. Iran J Pharma Res 14(3):757

20. Kaya M, Yıldııır Y, Çelik GY (2011) Synthesis and antimicrobial activities of novel bisacridine-1, 8-dione derivatives. Med Chem Res 20(3):293-299

21. Kawatkar SP, Keating TA, Olivier NB, Breen JN, Green OM, Guler SY, Hentemann MF, Loch JT, McKenzie AR, Newman JV (2014) Antibacterial inhibitors of gram-positive thymidylate kinase: structure-activity relationships and chiral preference of a new hydrophobic binding region. J Med Chem 57:4584-4597

22. Sogabe S, Masubuchi M, Sakata K, Fukami TA, Morikami K, Shiratori Y, Ebiike H, Kawasaki K, Aoki Y, Shimma N (2002) Crystal structures of candida albicans $\mathrm{N}$-myristoyltransferase with two distinct inhibitors. Chem Biol 9:1119-1128

23. Andrews JM (2010) BSAC methods for antimicrobial susceptibility testing. British Society for Antimicrobial Chemotherapy, Birmingham

24. Molecular Operating Environment (MOE). Version 2013.09, Montreal: Chemical Computing Group, Inc. 2017. http://www.chemcomp.com

25. Gilson MK, Liu T, Baitaluk M, Nicola G, Hwang L, Chong J (2016) BindingDB in 2015: a public database for medicinal chemistry, computational chemistry and systems pharmacology. Nucleic Acids Res 44(D1):D1045-D1053

\section{Submit your manuscript to a SpringerOpen ${ }^{\circ}$ journal and benefit from:}

- Convenient online submission

- Rigorous peer review

- Open access: articles freely available online

- High visibility within the field

- Retaining the copyright to your article

Submit your next manuscript at springeropen.com 